\title{
Adequação da prescrição de medicamentos na Atenção Primária à Saúde de Ribeirão Preto-SP: estudo transversal
}

\author{
Adequacy of drug prescriptions in the Primary Health Care of Ribeirão Preto-SP: \\ a cross-sectional study
}

\author{
Adecuación de las prescripciones de medicamentos en la Atención Primaria de Salud de \\ Ribeirão Preto-SP: estudio transversal
}

Maria Olívia Barboza Zanetti, Juliana Maldonado Marchetti, Regina Célia Garcia de Andrade

\section{Resumo}

Objetivo: Este estudo transversal visa analisar comparativamente as prescrições de medicamentos provenientes da atenção básica tradicional (Unidades Básicas de Saúde - UBS) com as da Estratégia de Saúde da Família (ESF). Métodos: Foram incluídas 1053 prescrições, alocadas em dois grupos: 932 provenientes de UBS e 121 da ESF. Tais prescrições foram analisadas de acordo com a adequação aos itens legalmente exigidos e aos indicadores de qualidade (presença de antimicrobianos, presença de injetáveis, uso da denominação oficial, uso da relação de medicamentos essenciais, média de medicamentos prescritos). Resultados: As prescrições da ESF se mostraram estatisticamente mais completas quanto à presença do endereço do prescritor (82,6\% UBS, 96,7\% ESF), à ausência de rasuras $(90,3 \%$ UBS, $96,7 \%$ ESF) e ao cumprimento dos aspectos legais exigidos referentes ao uso do medicamento, sendo eles: forma farmacêutica (70,7\% UBS, 80,2\% ESF), dose (70,9\% UBS, 79,3\% ESF), posologia (63,0\% UBS, $75,2 \%$ ESF), via de administração (58,3\% UBS, 83,5\% ESF) e duração do tratamento (76,9\% UBS, 92,6\% ESF). Os resultados dos indicadores de qualidade da prescrição se aproximaram nos dois modelos de atenção básica e estão de acordo com os valores recomendados, com exceção da média de medicamentos prescritos, a qual evidenciou uma tendência à polifarmácia no município (3,9 medicamentos por prescrição na UBS e 3,5 na ESF). Conclusão: Apesar dos resultados revelarem práticas inapropriadas na prescrição de medicamentos na Atenção Primária como um todo, há evidências de que as prescrições da ESF estão mais próximas ao padrão ideal, o que pode favorecer o Uso Racional de Medicamentos.

\section{Abstract}

Objective: This cross-sectional study aims to comparatively analyze drug prescriptions from the traditional basic health care service (Basic Health Units - BHU) with those from Family Health Strategy (FHS). Methods: A total of 1053 prescriptions were included, allocated in two groups: 932 from BHU and 121 from FHS. These prescriptions were analyzed according with compliance to legally required items and quality indicators (presence of antimicrobials, presence of injectable, use of the official name, use of the list of essential drugs, average of drugs prescribed). Results: The ESF prescriptions were more complete regarding the presence of the prescriber's address $(82.6 \% \mathrm{BHU}, 96.7 \%$ FHS), the absence of erasures and overwritten words (90.3\% BHU, 96.7\% FHS) and with compliance to legally required items related to use of drugs, being: pharmaceutical form (70.7\% BHU, 80.2\% FHS), dose (70.9\% BHU, $79.3 \% \mathrm{FHS})$, posology (63.0\% BHU, 75.2\% FHS), administration route (58.3\% BHU, 83.5\% FHS) and time of therapy $(76.9 \% \mathrm{BHU}$, $92.6 \% \mathrm{FHS}$ ). The results of the indicators of quality of prescription are approximated in both models of basic attention and according to the recommended values, with the exception of the average of drugs prescribed, which showed a tendency to polypharmacy at county ( 3.9 drugs per prescription at the BHU and 3.5 at the FHS). Conclusion: although the results reveal inappropriate practices in drug prescription at Primary Health Care services as a whole, there are evidence that prescriptions from FHS are closer to the ideal pattern, which may favor the Rational Use of Drugs.

Como citar: Zanetti MOB, Marchetti JM, Andrade RCG. Adequação da prescrição de medicamentos na Atenção Primária à Saúde de Ribeirão Preto-SP: estudo transversal. Rev Bras Med Fam Comunidade. 2017;12(39):1-11. http://dx.doi.org/10.5712/rbmfc12(39)1443
Palavras-chave:

Atenção Primária à Saúde Estratégia Saúde da Família Prescrições de Medicamentos

Keywords:

Primary Health Care Family Health Strategy Drug Prescriptions

Fonte de financiamento: declaram não haver.

Parecer CEP:

CEP/FCFRP $n^{\circ} .342$ (FCFRP/USP), aprovado em 16/06/2014

Conflito de interesses: declaram não haver. Procedência e revisão por pares: revisado por pares. Recebido em: 04/01/2017. Aprovado em: 12/01/2018. 


\section{Resumen}

Objetivo: Este estudio transversal tuvo como objetivo comparar prescripciones médicas de la atención primaria tradicional (Unidades Básicas de Salud - UBS) con las de la Estrategia de Salud Familiar (ESF). Métodos: Se incluyeron 1053 prescripciones, divididas en dos grupos: 932 de la UBS y 121 de la ESF. Estas prescripciones se analizaron de acuerdo con la adecuación de los ítems legalmente requeridos y a los indicadores de calidad (presencia de antimicrobianos, presencia de inyectables, uso de la denominación oficial, uso de la relación de medicamentos esenciales, número medio de los medicamentos prescritos). Resultados: Las prescripciones de la ESF eran estadísticamente más completas debido a la presencia de la dirección del prescriptor (82,6\% UBS, 96,7\% ESF), en la ausencia de tachaduras $(90,3 \%$ UBS, $96,7 \%$ ESF) y en el cumplimiento de los aspectos legales requeridos referentes al uso del medicamento, siendo ellos: forma farmacéutica (70,7\% UBS, 80,2\% ESF), dosis (70,9\% UBS, 79,3\% ESF), posología $(63,0 \%$ UBS, $75,2 \%$ ESF), vía de administración (58,3\% UBS, $83,5 \%$ ESF) y duración del tratamiento ( $76,9 \%$ UBS, 92,6\% ESF). LoS resultados de los indicadores de calidad de la prescripción fueron similares en los dos modelos de atención primaria y están de acuerdo con los valores recomendados, excepto el número medio de los medicamentos prescriptos, que mostró una tendencia a la polifarmacia en el municipio (3,9 medicamentos por prescripción en la UBS y 3,5 en la ESF). Conclusión: Aunque los resultados indican prácticas inadecuadas en la prescripción de medicamentos en atención primaria en general, existen evidencias de que las prescripciones de la ESF son más cercanas a los estándares, lo que puede favorecer el Uso Racional de Medicamentos.

\section{Introdução}

A Atenção Primária à Saúde (APS) é definida como a porta de entrada para o Sistema Único de Saúde (SUS) e como a ordenadora das práticas de atenção, ${ }^{1}$ embora ainda não tenha superado totalmente um modelo assistencial focado nas doenças que desconsidera o paciente como um ser integral que está inserido em um contexto familiar e sociodemográfico. ${ }^{2}$ Neste cenário, a Estratégia de Saúde da Família (ESF) foi implantada com o intuito de reorganizar o funcionamento da APS, em busca de universalidade, integralidade e equidade..$^{1,3}$ No país, a organização da APS é variável, sendo possível identificar no SUS dois modelos principais: atendimentos realizados conforme uma APS entendida como tradicional em Unidades Básicas de Saúde (UBS/UBDS); e atendimentos realizados de acordo com as diretrizes da ESF em Unidades de Saúde da Família (USF).

A proposta da ESF é reorganizar as condutas tradicionais da APS, de modo que a equipe de saúde passe a enxergar o usuário do sistema de modo integral, tendo a família como sua unidade de atuação e objetivo de atenção, reconhecendo os determinantes sociais que atuam sobre ela, comprometendo-se com a prevenção de doenças e com solução dos problemas de saúde da comunidade e realizando atividades de educação permanente em saúde. ${ }^{2-5}$ A ESF também se propõe a incorporar conceitos e práticas transformadoras, englobando a política nacional de medicamentos e, consequentemente, comprometendo-se com a promoção do uso racional de medicamentos (URM). ${ }^{5}$ Desta forma, se bem estruturada, a ESF pode ser um importante meio para que o URM se faça uma realidade.

Segundo a Organização Mundial da Saúde (OMS), "o uso racional de medicamentos requer que os pacientes recebam medicamentos apropriados para sua situação clínica, nas doses que satisfaçam as necessidades individuais, por um período adequado e ao menor custo possível para eles e sua comunidade". ${ }^{6}$ Portanto, a prescrição médica é um componente crucial para que ocorra o URM.

A política nacional de medicamentos considera os problemas de prescrição grandes obstáculos para o URM, pois a carência de informações importantes na prescrição pode favorecer o aparecimento de tratamentos ineficazes e prejuízos terapêuticos. ${ }^{5,7}$ No Brasil existem normatizações que visam regulamentar as informações contidas nas receitas, de modo a prevenir erros e fornecer valor jurídico 
ao documento. ${ }^{8}$ Entretanto, dentre as incontáveis prescrições de medicamentos elaboradas anualmente no SUS, grande parte não contém os requisitos legais mínimos exigidos. ${ }^{9} \mathrm{~A}$ análise de conformidade da prescrição com os determinantes exigidos por lei e com indicadores de qualidade colabora para uma análise introdutória da qualidade do tratamento, posto que revela erros que podem comprometer a sua segurança e efetividade. ${ }^{10-12}$

No cenário da APS, a ESF vem recebendo destaque e mostrando melhores resultados, ${ }^{4,13-16}$ no entanto, ainda não há estudos que analisem a prática da prescrição médica nesta estratégia. O projeto de transformar a atenção básica em um sistema centrado no usuário deve ter uma abordagem diferenciada também em relação ao processo de prescrição de medicamentos, tendo como propósito o URM.

O objetivo do presente estudo foi analisar comparativamente as prescrições de medicamentos provenientes da atenção básica tradicional com aquelas provindas da ESF, por meio da investigação da adequação das prescrições aos requisitos exigidos pela Lei no 5.991/73 e do cálculo dos indicadores de qualidade de prescrição propostos pela OMS.

\section{Métodos}

Trata-se de um estudo transversal realizado em Ribeirão Preto-SP, no período de setembro de 2014 a abril de 2015.

No início da coleta de dados desta pesquisa, a rede de atenção à saúde de Ribeirão Preto-SP contava com 41 estabelecimentos de APS em funcionamento, distribuídos por cinco Distritos de Saúde, sendo 28 UBS e 13 USF, com um total de 30 Equipes de Saúde da Família. O município ainda contava com 38 farmácias vinculadas à Secretaria Municipal de Saúde, proporcionando aos munícipes o acesso aos medicamentos do Componente Básico da Assistência Farmacêutica contidos na Relação Municipal de Medicamentos Essenciais (REMUME), que disponibilizava mais de 350 itens. $^{17}$

O tamanho amostral foi obtido considerando um plano estratificado. As Unidades de Saúde de Ribeirão Preto-SP foram agrupadas em oito estratos, de acordo com o Distrito de Saúde em que se localizam e com o modelo de atenção básica oferecido (UBS ou ESF). Considerou-se um coeficiente de confiança de 95\% e uma precisão absoluta de 3\% para a estimativa de proporção. Para maximização da variância, tal proporção foi considerada igual a 50\% em cada estrato, resultando em um tamanho amostral de 1.052 prescrições.

A alocação da amostra foi proporcional à média de consultas médicas/mês de cada estrato, totalizando 931 prescrições provenientes das Unidades Básicas de Saúde (UBS) e 121 da Estratégia de Saúde da Família (ESF). Duas Unidades de Saúde de cada estrato foram sorteadas para a coleta das prescrições. As UBS que contavam com equipes de agentes comunitários foram excluídas do sorteio por cacterizarem uma transição entre os modelos de atenção básica.

Foram incluídos no estudo usuários da APS com idade igual ou acima de 18 anos e que receberam uma prescrição emitida pelo médico de pelo menos um medicamento. Excluíram-se os indivíduos que não eram capazes de se comunicar adequadamente. Quando o usuário concordava em participar da pesquisa, era fornecido o Termo de Consentimento Livre e Esclarecido (TCLE), por meio do qual se formalizou a inclusão de todos os participantes, que autorizaram que sua prescrição fosse copiada. 
A análise do conteúdo das prescrições foi embasada nos critérios pré-estabelecidos pela Lei no $5.991 / 73,{ }^{8}$ sendo eles: estar legivelmente escrito em português e à tinta; apresentar o nome e endereço do usuário; utilizar a nomenclatura oficial do medicamento; conter forma farmacêutica, dose, quantidade total de medicamento a ser dispensado, posologia completa, via de administração e duração do tratamento; exibir a data de emissão; exibir nome, endereço, número de inscrição no conselho profissional e assinatura do prescritor. Verificou-se também a presença dos itens vedados, que podem induzir a erros, sendo eles: rasuras ou emendas e escrita abreviada. A posologia foi julgada como completa quando a prescrição apresentava informações de horário de tomada do medicamento e informações adicionais fundamentais para o URM (ex.: uso do medicamento com alimentos ou em jejum, dissolução em água, entre outros).

A avaliação da legibilidade de uma prescrição pode ser muito subjetiva e variar conforme a experiência do avaliador. Na tentativa de estabelecer um padrão mais homogêneo, adotou-se a classificação de legibilidade de Rosa et al. ${ }^{18}$ Cada palavra foi examinada e avaliada separadamente, de forma a evitar deduções pelo contexto, e as prescrições foram classificadas em legíveis, pouco legíveis ou ilegíveis. ${ }^{18}$

Considerou-se que o medicamento foi prescrito pela nomenclatura oficial quando era utilizada a Denominação Comum Brasileira (DCB) e, nos casos omissos, a Denominação Comum Internacional (DCl).

As prescrições de medicamentos ainda foram classificadas de acordo com o tipo de redação:

- Manuais: elaboradas manualmente pelo prescritor, a possibilidade de apresentarem itens omissos ou de serem ilegíveis é maior;

- Eletrônicas: digitadas pelo prescritor por meio de um sistema computadorizado, diminui dificuldades de leitura e ocorrência de erros;

- Mistas: uma parte é digitada e a outra parte é escrita manualmente pelo prescritor. ${ }^{19}$

Para a realização do cálculo dos indicadores de qualidade recomendados pela OMS, tomou-se como base o manual "How to investigate drug use in health facilities", ${ }^{12,20}$ conforme descrito a seguir:

- Número médio de medicamentos por prescrição = total de medicamentos prescritos/total de prescrições no período.

- Porcentagem de medicamentos prescritos pelo nome oficial = total de medicamentos com nome oficial/total de medicamentos, multiplicando-se por 100.

- Porcentagem de prescrições em que se prescrevem antimicrobianos = prescrições em que foi prescrito pelo menos um antimicrobiano/total de prescrições, multiplicando-se por 100 .

- Porcentagem de prescrições em que se prescrevem injetáveis = prescrições em que foi prescrito ao menos um injetável/total de prescrições, multiplicando-se por 100.

- Porcentagem de medicamentos prescritos que pertencem à Lista de Medicamentos Essenciais = total de medicamentos prescritos que figuram na lista/total de medicamentos prescritos, multiplicando-se por 100.

Adotou-se a REMUME vigente em 2014 como Lista de Medicamentos Essenciais. O uso concomitante de cinco ou mais medicamentos foi caracterizado como polifarmácia. ${ }^{21}$

As análises estatísticas foram realizadas por meio do programa Statistical Analysis System (SAS), considerando o nível de significância $\alpha=0,05$ e o intervalo de confiança=95\%. A condução do estudo foi aprovada, em 17 de junho de 2014, com parecer registrado no Sistema Nacional de Informação sobre Ética em Pesquisa envolvendo Seres Humanos (CAAE no 28445914.1.0000.5403). 


\section{Resultados}

No que se refere ao tipo de redação, 53\% $(n=558)$ do total de prescrições coletadas eram eletrônicas. Este valor é correspondente a 54,3\% $(n=506)$ das prescrições de UBS e $43 \%(n=52)$ das de ESF. Nenhuma prescrição eletrônica foi considerada ilegível ou pouco legível. As prescrições manuais equivalem a 41,1\% $(n=433)$ do total, perfazendo 39,4\% $(n=367)$ das prescrições de UBS e $54,5 \%(n=66)$ das de ESF. Três prescrições manuais de UBS foram escritas a lápis. O percentual restante $(5,9 \%, n=62)$ é referente ao tipo mista, sendo correspondente a 6,3\% $(n=59)$ das prescrições de UBS e 2,5\% (n=3) das de ESF.

Em relação ao índice de legibilidade, 3,6\% $(n=38)$ do total de prescrições foram classificadas como ilegíveis, sendo todas provindas de UBS. Nenhuma prescrição de ESF foi considerada ilegível, entretanto, $21,5 \%(n=26)$ foram classificadas como pouco legíveis. Esta frequência foi de $10,2 \%(n=95)$ nas prescrições de UBS.

Para o tratamento estatístico dos resultados, realizou-se testes exato de Fisher, visando verificar a relação das variáveis com o tipo de atendimento (UBS ou ESF) (Tabela 1). Com base nos valores $p$ obtidos, conclui-se que há evidências de que as variáveis "forma farmacêutica", "dose", "posologia completa", "via de administração", "duração do tratamento", "endereço do prescritor" e não apresentar "rasuras ou emendas" são mais completas nas prescrições de ESF. Entretanto, também há evidências de que os prescritores de UBS são mais aderentes ao modelo de prescrição eletrônica.

Tabela 1. Comparação entre o conteúdo das precrições médicas exigido por lei. Ribeirão Preto-SP, 2016.

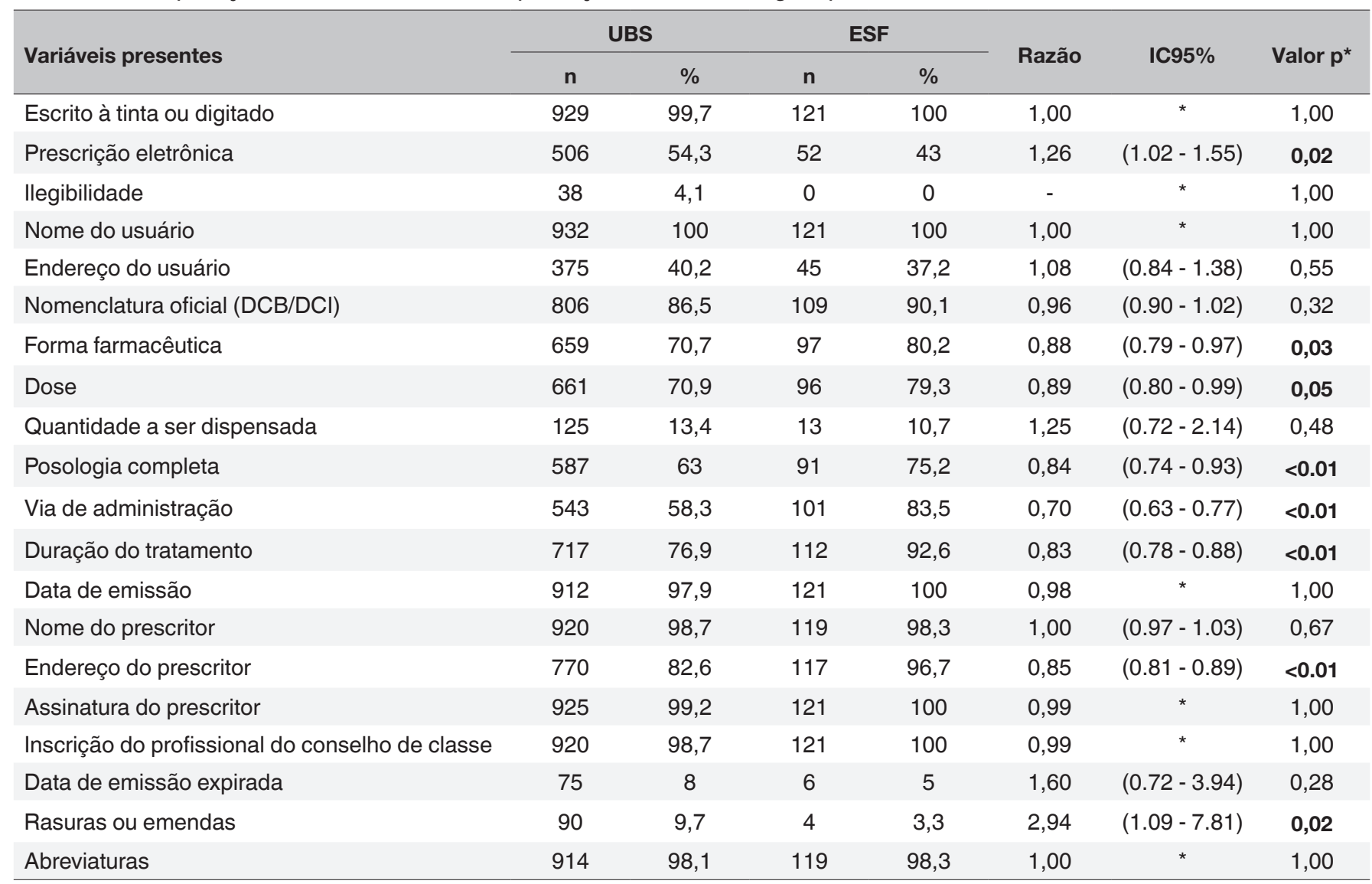

*Valores p obtidos de teste exato de Fisher. UBS: Unidade Básica de Saúde; ESF: Estratégia de Saúde da Família; IC95\%: Intervalo de confiança de 95\%. 
Na avaliação do item "posologia completa", nenhuma prescrição analisada apresentou uma informação adicional não preconizada na literatura médica ou nas bulas dos medicamentos.

De acordo com a OMS, um dos indicadores de qualidade da prescrição é a média de medicamentos prescritos por receita. Nas prescrições de UBS este valor foi de 3,9 (DP=2,8), variando de 1 a 20 medicamentos por prescrição. Nas precrições ESF, a média foi de 3,5 (DP=2,4), variando de 1 a 11 medicamentos. As frequências de prescrições contendo um, dois, três, quatro e cinco ou mais medicamentos estão expostas na Figura 1. No total, 32,6\% $(n=343)$ dos participantes encontravam-se submetidos à polifarmácia, sendo esta frequência superior nas Unidades Básicas de Saúde [33,3\% UBS ( $n=310) ; 27,3 \%$ ESF ( $n=33)]$.

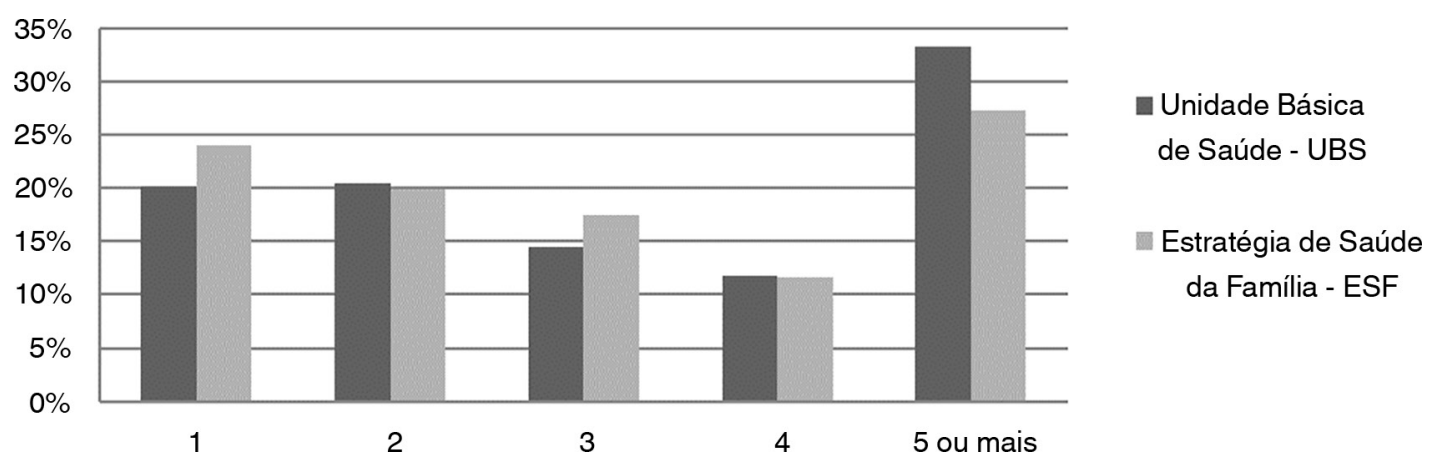

Figura 1. Comparação entre a frequência do número medicamentos prescritos. Ribeirão Preto-SP, 2016.

Os valores dos outros indicadores de qualidade da prescrição propostos pela OMS são apresentados na Tabela 2, a qual compara os resultados obtidos nos dois modelos de atenção básica com os valores de referência sugeridos pela OMS.

Tabela 2. Comparação dos resultados obtidos para os Indicadores da Qualidade da OMS. Ribeirão Preto-SP, 2016.

\begin{tabular}{lccccc}
\hline & \multicolumn{2}{c}{ UBS } & \multicolumn{2}{c}{ ESF } & Valores de referência OMS (\%) \\
\cline { 2 - 5 } Indicadores da OMS & $\%$ & $\mathbf{n}$ & $\%$ & $\mathbf{n}$ & 30,0 \\
\hline Prescrições contendo antimicrobianos & 11,4 & 106 & 10,7 & 13 & 10,0 \\
Prescrições contendo injetáveis & 9,7 & 90 & 3,3 & 4 & 100,0 \\
Medicamentos prescritos pela denominação oficial & 92,2 & 859 & 92,4 & 112 & 100,0 \\
Medicamentos prescritos que constam na REMUME & 91,1 & 849 & 91,0 & 110 & \\
\hline
\end{tabular}

UBS: Unidade Básica de Saúde; ESF: Estratégia de Saúde da Família; OMS: Organização Mundial da Saúde.

\section{Discussão}

A dispensação de medicamentos é efetuada a partir de prescrições manuais, eletrônicas ou mistas. Dentre estas, a manual é a principal responsável pela problemática das prescrições incompletas, ilegíveis ou pouco legíveis, ${ }^{19}$ sendo que os pesquisadores Rosa e Perini ${ }^{22}$ constataram que as prescrições manuais apresentam 6,3 vezes mais chance de apresentarem problemas e as mistas um risco 3,5 vezes maior. Portanto, uma medida interessante para diminuir os erros de prescrição é o emprego da prescrição eletrônica.

O modelo de prescrição eletrônica foi adotado pela SMS-RP e está disponível em todas as Unidades de Saúde desde 2013. O programa preenche automaticamente as informações do usuário, do prescritor e a data de emissão, além de não permitir a emissão caso os campos "nome do medicamento", "dose", 
"forma farmacêutica", "unidade" e "via de administração" não estejam completos. O uso desta ferramenta evitaria a ausência de algumas informações importantes nas prescrições e a ilegibilidade, porém este estudo atesta que muitos prescritores ainda não aderiram a esta ferramenta. Na ESF a prescrição manual ainda é a forma predominante, o que justifica o número maior de prescrições pouco legíveis encontrado neste modelo de atenção básica.

O nome do usuário estava presente em todas as prescrições coletadas, entretanto, o endereço foi um item faltante em mais da metade das prescrições dos dois modelos de atenção básica. A prescrição de medicamentos é um documento pessoal e intransferível, portanto, deve conter informações específicas sobre o usuário para a qual foi emitida. A falta de identificação pode acarretar no comprometimento do contato do farmacêutico após a dispensação em caso de necessidade. ${ }^{19}$

As informações sobre o prescritor também estavam contidas na maioria das prescrições de ambos os modelos de atenção básica, exceto o endereço. A presença destes itens constitui uma possibilidade concreta de responsabilização em casos de erro médico. Tais informações ainda permitem o contato do usuário e do farmacêutico com o prescritor para esclarecimento de dúvidas. Ademais, a falta de informações sobre o prescritor e sobre o usuário facilita a ocorrência de fraudes e falsificações, promovendo o uso abusivo ou ilícito de medicamentos. ${ }^{19}$

As prescrições emitidas pela ESF apresentaram superioridade na presença das variáveis legais referentes ao medicamento: forma farmacêutica, dose, posologia completa, via de administração e duração do tratamento. A ausência de forma farmacêutica, dose ou via de administração em uma prescrição dificulta a dispensação e pode favorecer a troca do medicamento indicado pelo prescritor e erros de administração. ${ }^{9,23} \mathrm{~A}$ falta ou incompletude da posologia pode gerar dúvidas sobre o tratamento e acarretar prejuízos farmacoterapêuticos, uma vez que omite informações importantes para o uso correto do medicamento em questão. ${ }^{24}$ Quanto ao item "duração do tratamento", a sua omissão pode acarretar a interrupção do tratamento antes do período adequado.

O destaque da ESF no tocante à presença destas variáveis é uma importante contribuição para diminuir os erros de medicação e promover o URM. Convém reiterar que a ausência destes itens é um tipo de erro bastante preocupante, pois afeta diretamente a compreensão do usuário e pode acarretar problemas na utilização do medicamento, prejuízo terapêutico, tratamentos inefetivos e desperdícios. ${ }^{10,19}$

A expiração da data de emissão, presente em ambos os modelos de atenção básica, pode ser um indicativo da dificuldade em agendar um retorno ao médico.

A alta incidência de escrita abreviada nas prescrições dos dois modelos é preocupante. A legislação exige que as prescrições de medicamentos não contenham nenhum tipo de abreviatura, sigla ou símbolo, dado que diversos estudos atestaram que abreviações podem causar dúvidas e interpretações errôneas na dispensação e utilização do medicamento. ${ }^{19,25,26}$ Entretanto, é evidente que, por economizar tempo e simplificar a redação, a emissão de prescrições abreviadas ainda é uma realidade que deve ser comedida.

Em relação aos indicadores de qualidade da prescrição propostos pela OMS, os resultados se aproximam nos dois modelos de atenção básica e estão de acordo com os valores recomendados, com exceção da média de medicamentos prescritos por prescrição. 
Tal média está acima do número recomendado pela OMS, que considera 1,3 a 2,2 medicamentos por prescrição um resultado sem tendência à polifarmácia. ${ }^{12}$ Portanto, apesar de um pouco inferior na ESF, em ambos os modelos de atenção há uma forte tendência à polifarmácia. Estudo semelhante realizado pelas pesquisadoras Santos e Nitrini também no munícipio de Ribeirão Preto, no ano de 2004, encontrou uma média de 2,4 medicamentos por prescrição. ${ }^{20}$ Isto sugere que, em um período de dez anos, a preocupação dos prescritores com a polifarmácia pode ter diminuído. A média encontrada em outros estudos efetuados em municípios brasileiros variou entre 2,0 e 2,8 medicamentos por prescrição, confirmando a elevação obtida neste estudo. ${ }^{27-31}$

Um estudo realizado na APS de Vitória da Conquista-BA analisou 350 prescrições e em apenas 8,6\% delas havia cinco ou mais medicamentos prescritos. ${ }^{29}$ Este valor é muito inferior ao aqui encontrado, o que sinaliza que polifarmácia é uma realidade no município de Ribeirão Preto-SP.

Apesar da quantidade de medicamentos prescritos descrita já ser alta, é possível que ela seja ainda maior, pois os dados coletados referem-se aos medicamentos contidos na prescrição emitida pelo médico que o usuário portava no momento da abordagem, o que não descarta a possibilidade de haver outros medicamentos em uso, indicados por outro prescritor. Este fato é alarmante, porque a polifarmácia associada a outras situações, como reações adversas e interações medicamentosas, pode tornar o tratamento demasiadamente complexo e prejudicar a adesão. ${ }^{21,30}$ Entretanto, é preciso enfatizar que em muitos casos a polifarmácia é inevitável, como em pacientes com problemas de saúde graves ou muitas comorbidades.

A porcentagem de receitas em que se prescreveram antimicrobianos obtida neste estudo está de acordo com o recomendado pela International Network for the Rational Use of Drug (INRUD), indicando valores de até $30 \%$ como aceitáveis. ${ }^{30,32}$ Quando comparada ao valor de $21,3 \%$, que era prescrito há mais de dez anos no município ${ }^{20}$ e aos valores de $19 \%$ encontrado no estudo de Souza et al. ${ }^{31}$ e de $22 \%$ obtido por Girotto e Silva ${ }^{27}$ em outros municípios brasileiros, a frequência de antimicrobianos prescritos nos dois modelos de atenção básica foi bastante reduzida. Isto pode estar associado a fatores climáticos do período do ano em que a coleta de dados sucedeu, mas também pode relacionar-se com o aumento da preocupação dos prescritores com o uso indiscriminado destes medicamentos e a geração de bactérias resistentes.

Quanto à prescrição de injetáveis, os valores estão condizentes com o recomendado pela OMS, que considera frequências abaixo de 10\% como aceitáveis, e com o encontrado anteriormente em Ribeirão Preto $(8,3 \%)^{20}$. Em outros estudos brasileiros, a frequência de prescrições da APS contendo injetáveis se aproximou ao valor obtido nas prescrições de UBS, variando entre 2,9 e 7\%.27,28,30,31

Merece destaque a porcentagem de medicamentos prescritos pelo nome oficial, que foi alta em ambos os modelos de atenção básica e muito maior do que a relatada em $2004(30,6 \%) .{ }^{20} \mathrm{O}$ uso da denominação oficial ainda foi alto se comparado a outros municípios, nos quais a frequência oscilou entre 70,2 e $86,8 \%{ }^{27,28,30,31}$ A OMS recomenda que seja adotada a nomenclatura oficial do medicamento em todas as prescrições geradas (100\%), porque facilita a educação e a informação da população. No Brasil, a Lei no 9.787 de 1999 regulamenta esta prática no âmbito do SUS. ${ }^{33}$

Tendo em vista que o acesso da população àqueles medicamentos considerados essenciais é um dos objetivo principais definidos pela política nacional de medicamentos, o município de Ribeirão Preto está em concordância com o estabelecido, pois verificou-se que a maior parte dos medicamentos prescritos nos dois modelos do atenção básica integravam a REMUME. 
No ano de 2004, Santos e Nitrini encontraram uma porcentagem inferior à atual $(83,4 \%),{ }^{20}$ o que aponta que a REMUME está mais adequada ao perfil epidemiológico da população local, que os prescritores entenderam a importância de aderir à lista e que atualmente o acesso da população aos medicamentos está mais favorecido. As frequências obtidas neste estudo ainda superam os valores de outros municípios, que oscilam entre $58,4 \%$ e $85,3 \%, 27,28,30$ e se assemelham ao valor obtido no sul do Estado de Santa Catarina $(91,5 \%) .{ }^{31}$ Entretanto, pelo fato da maioria das prescrições terem sido coletadas na fila das farmácias, salienta-se que, no momento da consulta, o prescritor pode ter avisado o usuário que o medicamento não estaria disponível na farmácia das Unidades e que deveria ser comprado. Nesse sentido, este dado pode estar superestimado.

Em geral, as prescrições provenientes da ESF mostraram-se mais completas quanto ao cumprimento dos aspectos legais exigidos referentes ao uso do medicamento, à presença do endereço do prescritor e à ausência de rasuras. Portanto, há evidências de que as prescrições da ESF estão mais próximas ao padrão ideal. Considerando que a política nacional de medicamentos afirma que a prescrição de medicamentos impacta diretamente sobre o seu uso racional, ${ }^{34}$ as prescrições da ESF de Ribeirão Preto-SP revelaram-se mais favoráveis ao URM.

Uma vez que a análise de prescrições médicas não se restringe ao cumprimento da legislação, algumas limitações deste estudo devem ser apontadas. O quadro clínico do usuário do serviço e os aspectos da decisão terapêutica não foram alvo de análise, desta forma, tal tipo de erro de prescrição não foi avaliado. Do mesmo modo, a situação de polifarmácia obtida não pode ser julgada como necessária ou não. Outra limitação deve-se ao fato de que os medicamentos sujeitos a controle especial e os antimicrobianos não foram objetos de verificação quanto ao cumprimento de suas legislações específicas.

\section{Conclusão}

O estudo propiciou identificar inadequações nas prescrições médicas elaboradas pela APS de Ribeirão Preto-SP. Os resultados obtidos revelam práticas inapropriadas na prescrição de medicamentos que são desfavoráveis ao URM em ambos os modelos de atenção básica, como a ausência de elementos fundamentais da prescrição e exigidos por lei, a não adesão ao modelo de prescrição eletrônica, o uso abusivo da escrita abreviada e a média de medicamentos prescritos superior a outros municípios brasileiros. É necessário orientar o prescritor para que ele enxergue a prescrição como uma aliada na farmacoterapia, que assegura o URM. Entretanto, o presente estudo encontrou indícios de que o processo de prescrição racional de medicamentos está mais bem estabelecido entre os prescritores da ESF, ainda que não esteja no nível ideal.

\section{Referências}

1. Brasil. Ministério da Saúde. Portaria no 648, de 28 de março de 2006. Aprova a Política Nacional de Atenção Básica, estabelecendo a revisão de diretrizes e normas para a organização da Atenção Básica para o Programa Saúde da Família (ESF) e o Programa Agentes Comunitários de Saúde. Brasília: Diário Oficial da União; 2006.

2. Goldbaum M, Gianini RJ, Novaes HMD, César CLG. Utilização de serviços de saúde em áreas cobertas pelo programa saúde da família (Qualis) no Município de São Paulo. Rev Saúde Pública. 2005;39(1):90-9. DOI: http://dx.doi.org/10.1590/S0034-89102005000100012

3. Brasil. Ministério da Saúde. Manual para a organização da Atenção Básica. 3aㅡ ed. Brasília: Ministério da Saúde; 1999. 
4. Arantes LJ, Shimizu HE, Merchán-Hamann E. Contribuições e desafios da Estratégia Saúde da Família na Atenção Primária à Saúde no Brasil: revisão da literatura. Ciênc Saúde Coletiva. 2016;21(5):1499-510. DOI: http://dx.doi.org/10.1590/1413-81232015215.19602015

5. Oliveira LCF, Assis MMA, Barboni AR. Assistência Farmacêutica no Sistema Único de Saúde: da Política Nacional de Medicamentos à Atenção Básica à Saúde. Ciênc Saúde Coletiva. 2010;15(Suppl 3):3561-7. DOI: http://dx.doi.org/10.1590/S1413-81232010000900031

6. World Health Organization (WHO). The Rational Use of Drugs: Report of the Conference of Experts. Geneva: World Health Organization; 1987.

7. Arrais PSD, Barreto ML, Coelho HLL. Aspectos dos processos de prescrição e dispensação de medicamentos na percepção do paciente: estudo de base populacional em Fortaleza, Ceará, Brasil. Cad Saúde Pública.2007;23(4):927-37. DOI: http://dx.doi.org/10.1590/S0102$311 \times 2007000400020$

8. Brasil. Lei no 5.991, de 17 de dezembro de 1973. Dispõe sobre o controle sanitário do comércio de drogas, medicamentos, insumos farmacêuticos e correlatos, e dá outras providências. Brasília: Diário Oficial da República Federativa do Brasil; 1973.

9. Lyra-Júnior DP, Prado MCTA, Abriata JP, Pelá IR. Recetas médicas como causantes de riesgo de problemas relacionados com medicamentos. Seguim Farm. 2004;2(2):86-96.

10. Mastroianni PC. Análise dos aspectos legais das prescrições de medicamentos. Rev Ciênc Farm Básica Apl. 2009;30(2):173-6.

11. Benjamin DM. Reducing medication errors and increasing patient safety: case studies in clinical pharmacology. J Clin Pharmacol. 2003;43(7):768-83. DOI: http://dx.doi.org/10.1177/0091270003254794

12. World Health Organization (WHO). How to investigate drug use in health facilities: selected drug use indicators. Geneva: World Health Organization; 1993.

13. Gragnolati M, Lindelow M, Couttolenc B. Twenty Years of Health System Reform in Brazil: An Assessment of the Sistema Único de Saúde. 1st ed. Washington: World Bank; 2013.

14. Organização Mundial da Saúde - Comissão para os Determinantes Sociais da Saúde (CDSS-OMS). Redução das desigualdades no período de uma geração: igualdade na saúde através da ação sobre os seus determinantes sociais. Relatório Final da Comissão para os Determinantes Sociais da Saúde. 1a ed. Lisboa: Organização Mundial da Saúde; 2010.

15. Aquino R, de Oliveira NF, Barreto ML. Impact of the family health program on infant mortality in Brazilian municipalities. Am J Public Health. 2009;99(1):87-93. DOI: http://dx.doi.org/10.2105/AJPH.2007.127480

16. Dourado I, Oliveira VB, Aquino R, Bonolo P, Lima-Costa MF, Medina MG, et al. Trends in primary health care-sensitive conditions in Brazil: the role of the Family Health Program (Project ICSAP-Brazil). Med Care. 2011;49(6):577-84. DOI: http://dx.doi.org/10.1097/ MLR.0b013e31820fc39f

17. Prefeitura Municipal de Ribeirão Preto. Secretaria Municipal da Saúde.Plano Municipal de Saúde:2014-2017. Ribeirão Preto: Secretaria Municipal da Saúde -Ribeirão Preto; 2013.

18. Rosa MB, Perini E, Anacleto TA, Neiva HM, Bogutchi T. Erros na prescrição hospitalar de medicamentos potencialmente perigosos. Rev Saúde Pública. 2009;43(3):490-8. DOI: http://dx.doi.org/10.1590/S0034-89102009005000028

19. Silva SM. Avaliação de prescrições em farmácia comunitária universitária. [dissertação]. Araraquara: Universidade Estadual Paulista Júlio de Mesquita Filho; 2012.

20. Santos V, Nitrini SMOO. Indicadores do uso de medicamentos prescritos e de assistência ao paciente de serviços de saúde. Rev Saúde Pública. 2004;38(6):819-34. DOI: http://dx.doi.org/10.1590/S0034-89102004000600010

21. Lucchetti G, Granero AL, Pires SL, Gorzoni ML. Fatores associados à polifarmácia em idosos institucionalizados. Rev Bras Geriatr Gerontol. 2010;13(1):51-8. DOI: http://dx.doi.org/10.1590/S1809-98232010000100006

22. Rosa MB, Perini E. Erros de medicação: quem foi? Rev Assoc Med Bras. 2003;49(3):335-41. DOI: http://dx.doi.org/10.1590/S010442302003000300041

23. Portela AS, Simões MOS, Fook SML, Montenegro Neto AN, Silva PCD. Prescrição médica: orientações adequadas para o uso de medicamentos? Ciênc Saúde Coletiva. 2010;15(Suppl 3):3523-8. DOI: http://dx.doi.org/10.1590/S1413-81232010000900027

24. Fröhlich SE, Dal Pizzol TS, Mengue SS. Instrumento para avaliação do nível de conhecimento da prescrição na atenção primária. Rev Saúde Pública. 2010;44(6):1046-54. DOI: http://dx.doi.org/10.1590/S0034-89102010000600009

25. Ev LS, Guimarães AG, Castro VS. Avaliação das Prescrições Dispensadas em uma Unidade Básica de Saúde do Município de Ouro Preto, Minas Gerais, Brasil. Lat Am J Pharm. 2008;27(4):543-7. 
26. Instituto para Práticas Seguras no Uso de Medicamentos do Brasil (ISMP Brasil). Erros de medicação associados a abreviaturas, siglas e símbolos. [Internet]. Belo Horizonte: ISMP Brasil; 2016. [acesso 2016 Nov 20]. Disponível em: http://www.boletimismpbrasil.org/boletins/ pdfs/boletim_ISMP_31.pdf

27. Girotto E, Silva PV. A prescrição de medicamentos em um município do Norte do Paraná. Rev Bras Epidemiol. 2006;9(2):226-34. DOI: http://dx.doi.org/10.1590/S1415-790X2006000200009

28. Laste G, Torres ILS, Deitos A, Souza AC, Souza A, Kauffmann C, et al. Análise de prescrições médicas dispensadas em farmácia no Sistema Único de Saúde. Rev HCPA. 2013;33(1):15-25.

29. Leão DFL, Moura CS, Medeiros DS. Avaliação de interações medicamentosas potenciais em prescrições da atenção primária de Vitória da Conquista (BA), Brasil. Ciênc Saúde Coletiva. 2014;19(1):311-8. DOI: http://dx.doi.org/10.1590/1413-81232014191.2124

30. Portela AS, Silva PCD, Simões MOS, Medeiros ACD, Montenegro-Neto AN. Indicadores de prescrição e de cuidado ao paciente na atenção básica do município de Esperança, Paraíba, 2007. Epidemiol Serv Saúde. 2012;21(2):341-50. DOI: http://dx.doi.org/10.5123/ S1679-49742012000200017

31. Souza JM, Vinholes ER, Trauthman SC, Galato D. Avaliação dos indicadores de prescrição e da demanda atendida de medicamentos no Sistema Único de Saúde de um município do Sul do Estado de Santa Catarina. Rev Ciênc Farm Básica Apl. 2012;33(1):107-13.

32. Louro E, Romano-Lieber NS, Ribeiro E. Eventos adversos a antibióticos em pacientes internados em um hospital universitário. Rev Saúde Pública. 2007;41(6):1042-8. DOI: http://dx.doi.org/10.1590/S0034-89102006005000049

33. Brasil. Lei no 9.787, de 10 de fevereiro de 1999. Altera a Lei no 6.360, de 23 de setembro de 1976, que dispõe sobre a vigilância sanitária, estabelece o medicamento genérico, dispõe sobre a utilização de nomes genéricos em produtos farmacêuticos e dá outras providências Brasília: Diário Oficial da União; 1999.

34. Brasil. Conselho Nacional de Secretários da Saúde. Assistência Farmacêutica no SUS. 1ํa ed. Brasília: CONASS; 2007.

Departamento de Ciências Farmacêuticas, Faculdade de Ciências Farmacêuticas de Ribeirão Preto, Universidade de São Paulo (FCFRP/USP). Ribeirão Preto, SP, Brasil. maria.zanetti@usp.br (Autora correspondente); jmarchet@usp.br; randrade@usp.br 